\title{
Recovery of Absolute Absorption Line Shapes in Tunable Diode Laser Spectroscopy using External Amplitude Modulation with Balanced Detection
}

\author{
James R.P. Bain, Michael Lengden, George Stewart and Walter Johnstone
}

\begin{abstract}
Accurate recovery of an absorption lineshape is important in many industrial applications for simultaneous measurement of gas concentration and pressure or temperature. Here we demonstrate a method, based on a modification to the Hobbs balanced receiver circuit, for background signal nulling when external amplitude modulation of the laser output is used. Compared with direct or non-nulled detection techniques, we demonstrate that the method significantly improves the signal to noise ratio to a level comparable to that of conventional second harmonic wavelength modulation spectroscopy. Most importantly, normalisation and recovery of the lineshape is straightforward and immune to the difficulties that afflict lineshape recovery with conventional wavelength modulation spectroscopy.
\end{abstract}

Index Terms-Balanced detection, absorption line shape recovery, diode laser spectroscopy, gas sensors, industrial and environmental monitoring, wavelength modulation spectroscopy (WMS).

\section{INTRODUCTION}

$\mathrm{N}$ EAR-INFRARED tunable diode laser spectroscopy (TDLS) has become a widely used method for gas measurement in industrial, environmental and safety applications. There are several molecules of interest that exhibit favourable overtone absorption spectra in the $1-2 \mu \mathrm{m}$ region which are observable using the robust and low-cost optoelectronic hardware developed for the telecommunications market. Optical fibre networks also enable distributed or remote sensing for gas measurements in the harsh environments that are of interest in this study [1, 2].

Direct detection of absorption line shapes can be recovered simply by ramping the drive current of a diode laser source to sweep the laser output frequency across a target absorption

This paragraph of the first footnote will contain the date on which you submitted your paper for review.

J. R. P. Bain was with the Electronic and Electrical Engineering Department, University of Strathclyde, Glasgow, Scotland, G1 1XW and is now with M SQUARED LASERS LTD, West of Scotland Science Park, Glasgow, Scotland, G20 0SP (e-mail: james.bain@M2lasers.com).

M. Lengden, G. Stewart and W. Johnstone are all with the Electronic and Electrical Engineering Department, University of Strathclyde, Glasgow, Scotland, G1 1XW (e-mail:michael.lengden@strath.ac.uk) feature. The change in detected transmission yields an absorption line shape based on the Beer-Lambert law for optical transmission from which direct measurements of species concentration, pressure, temperature or velocity can be obtained. The accuracy of the recovered line shape is dependent upon normalisation to a suitable reference signal based upon either a measurement in the absence of the absorbing species or from fitting to non-absorbing regions in the vicinity of the target absorption line. The signal processing required to extract gas information from the recovered line shape, for instance from a theoretical leastsquares fit, is relatively straightforward and robust. Hence direct detection is appropriate for long-term field deployment.

To achieve higher sensitivity measurements a wellestablished alternative to direct detection is to include a high frequency current dither on the laser current ramp for wavelength modulation spectroscopy (WMS) [3-10]. The recovered high frequency signal can be demodulated with a lock-in amplifier which can greatly improve the signal-tonoise ratio and hence overall sensitivity, as the high operating frequency will reduce laser and 1/f noise whilst the detection bandwidth is significantly reduced by the phase sensitive detection. A common implementation of this technique recovers the second harmonic signal at twice the modulation frequency which is advantageous for gain and sensitivity since the second harmonic has a (nominally) zero background level (as opposed to the high background of the fundamental $1^{\text {st }}$ harmonic signal). A zero baseline also provides greater flexibility in the choice of spectral features where adjacent absorption line overlap would compromise a baseline fit. Gas information can be recovered from the second harmonic line shapes from calibration curves or from full system characterisations where certain gas parameters such as pressure or temperature are known or controlled [9, 10]. Unfortunately, however, the recovered second harmonic line shape is inextricably linked to the gas state by the dependence of the line shape on the modulation index, $m=\delta v / \gamma$, which relates the extent of the frequency modulation, $\delta v$, to the absorption half-width, $\gamma$. For many harsh environments in industrial applications the pressure or temperature could be unknown or varying, therefore creating uncertainty in the absorption half-width, reducing the suitability of these well- 
established techniques for long-term sensor deployment in harsh industrial environments.

Recent studies by the authors have examined the recovery of absolute absorption line shapes from wavelength-modulated signals using the residual amplitude modulation (RAM) signals that occur at the fundamental frequency [5 - 11]. Two distinct methods have been described for isolating the absorption-dependent amplitude-modulated component of the recovered first harmonic signal from the frequency-modulated signals observed in conventional TDLS with WMS [5, 6]. Subsequent studies showed that these techniques can provide higher sensitivities by removing the RAM background using an optical delay line and by use of high modulation indices along with a correction procedure for the associated line shape distortion that occurs in the recovered signals at high modulation indices [9-11]. These two solutions can significantly improve the overall sensitivity of the techniques, especially when used together. However the increased complexity of signal recovery makes practical deployment more challenging.

The Hanson Group, from Stanford University, have developed an $\mathrm{nf} / 1 \mathrm{f}$ calibration-free TDLS-WM method to obtain concentration, pressure and temperature information in industrial environments [12-14]. In this methodology, the $1 f$ RAM is used to normalise an $n$th harmonic signal, with the resulting data being fitted to a model using a sum of squared error algorithm for the targeted gas parameters. We have also applied the $2 \mathrm{f} / 1 \mathrm{f}$ approach for our research on the monitoring of gas turbine exhaust species [15] because, in this case, the presence of overlapping absorption lines precludes obtaining a zero baseline for the direct approach as proposed here. However, there are a number of experimental parameters that have to be measured prior to, or during, process measurements making signal processing more complex than with a direct intensity modulated signal.

In this work a method for recovering absolute absorption line shapes from noisy environments with high sensitivity is proposed, based on pure amplitude modulation from an external lithium niobate amplitude modulator. In principle the system is analogous to early implementations of TDLS with phase sensitive detection which utilised optical choppers to modulate a free space beam. A typical optical chopper can modulate up to a few kilohertz which would not be suitable for many of the applications being investigated in this study. Furthermore, the ease of incorporation of integrated fibre modulators into single-mode fibre networks allows remote access in challenging application areas, making this methodology ideal for deployment in harsh environments. In addition to avoiding the requirement for modulation of a collimated free-space beam, the potential frequency of modulation extends into the gigahertz range which goes beyond the limiting bandwidth of readily available lock-in amplifiers. Recent studies by the authors into TDLS for aero engine exhaust plume measurements have shown that noise from mechanical vibrations and in-plume beam steering extends to hundreds of kilohertz which is also well beyond the range of mechanical choppers.
The extent of the amplitude modulation provided by the external modulators, i.e. from zero to $100 \%$, is significantly higher than the amplitude modulation achievable by the current modulation of DFB lasers applying AM-TDLS techniques as it is free from the associated distortion terms [5]. Additionally, the absence of the simultaneous frequency modulation with DFB lasers means that the recovered line shape represents the true line shape regardless of the modulation amplitude. For high sensitivity measurements, pure amplitude modulation greatly simplifies the signal processing compared with standard WMS techniques, since the DC output from the lock-in amplifier can be used in exactly the same way for normalisation as for direct detection. Under ideal laboratory conditions, the output from the demodulated external AM signal is very similar to the direct detection output but in a noisy field-deployed system the high bandwidth direct signal would be susceptible to noise at all frequencies within the photodiode bandwidth whereas use of the AM signal retains the same noise reduction benefits of standard WMS though the use of the lock-in amplifier. For many applications where the target absorption lines exhibit line strengths that provide sufficient SNRs over the required absorption path this method can be used in the simple form described above. For weakly absorbing transitions however, the achievable SNR may be inadequate for high accuracy measurements. A further improvement in sensitivity can be achieved by nulling the background AM signal such as by optical nulling as demonstrated previously with the fibre delay line [11]. However a limitation of this technique is the restriction in modulation frequency imposed by the length of the fibre delay line. It would be desirable to have some flexibility in the choice of modulation frequency so that the system could be adjusted to changes in the noise frequency spectrum if necessary.

In this work, we demonstrate an alternative solution to opticalnulling using a simple modification to the dual-beam noise canceller circuit first introduced by Hobbs [16-19]. This detection system enables the background AM signals to be removed in the receiver (Hobbs) circuit, allowing the lock-in amplifier to be operated with a gain that can be as much as a factor of 40 times higher than in the non-nulled case. (Note that for a typical lock-in amplifier, such as the Stanford SR830 DSP lock-in, the overall gain, input to output, is adjusted through the sensitivity setting and is related to the sensitivity setting by: Gain $=10 \mathrm{~V} /$ Sensitivity where $10 \mathrm{~V}$ DC is the full scale output). Here we show that the background nulling and common mode noise cancellation improves the SNR to a level comparable with conventional second harmonic WMS yet the detection procedure and signal processing required are much simpler and independent of any instrumentation scaling factors, system characterisation or specific local gas parameters. 


\section{Pure Amplitude Modulation With Background NULLING}

An absorbing species will cause a wavelength-dependent reduction in transmission based on the Beer-Lambert law, $I_{\text {out }}(v)=I_{\text {in }}(v) e^{-\alpha(v) C l}$. The recovered lock-in amplifier output from a purely amplitude modulated input signal will be analogous to a direct detection output with the absorption and background signals being recovered in proportion to the transmitted optical power. The current-power characteristics of typical laser diodes will create a significant change in peak to peak optical power over the current range required to sweep across one or several absorption features. This change in power associated with the current sawtooth will lead to a photodiode output voltage that will dominate the dynamic range of the lock-in amplifier, requiring a reduced gain setting to avoid saturation of the lock-in output.

Removing the background signal to leave the absorption signal on a near-zero background allows the gain of the lockin amplifier to be significantly increased without overloading the output. The balanced detector used to remove the background prior to demodulation is based on the Hobbs autobalanced noise canceller circuit, as shown in Figure 1, which cancels common-mode noise to near-shot noise levels [16]. In normal operation this circuit recovers the direct absorption signal from the DC feedback signal at the log ratio output in Figure 1, at a rate well within the bandwidth of the integrator circuitry. With wavelength modulation of a DFB source, harmonic signals may be recovered from the linear output connected to a lock-in amplifier, yielding harmonic line shapes with the RAM background removed as common-mode noise, provided that the high frequency modulation sits outside the integrator bandwidth.

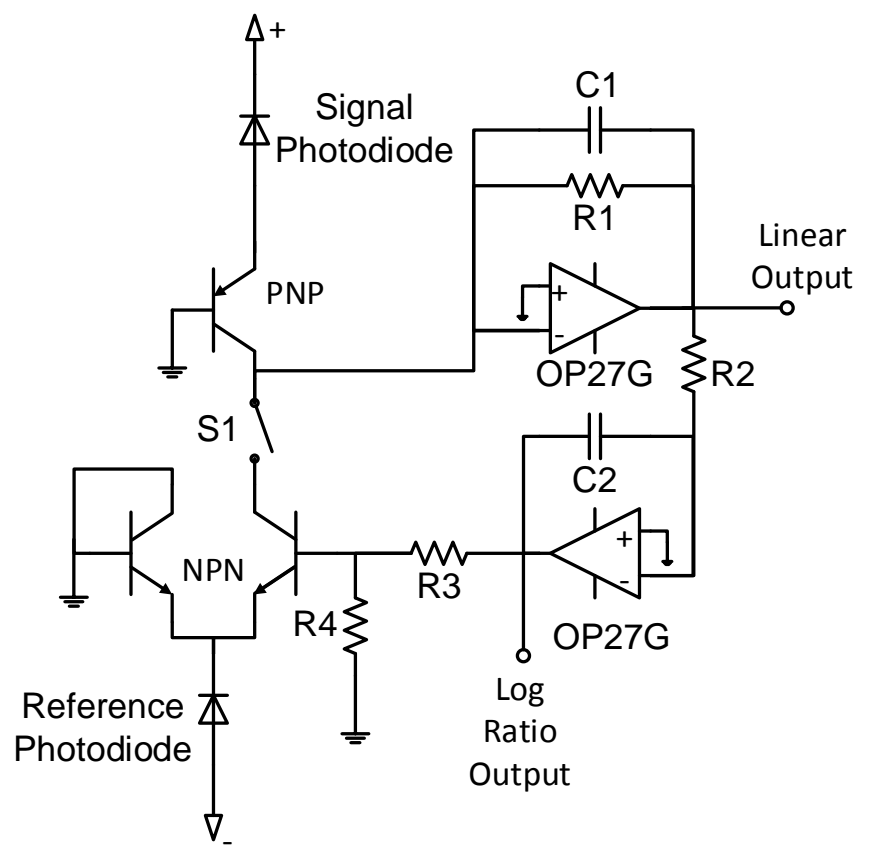

Fig. 1. Hobbs auto-balanced receiver circuit
In this study the intention is to recover the absorptiondependent AM signal from the linear output with the background AM nulled. Normal use of the Hobbs circuit is unsuitable because the AM absorption signal scales exactly with the DC absorption signal and the autobalance operation of the circuit (through the feedback loop) adjusts the base voltage to null the linear output for DC and hence for the AM absorption signal. To solve this problem the feedback bandwidth of the integrator is reduced significantly, through careful choice of resistor and capacitor values, until the integrator frequency response is well below the sawtooth current ramp frequency. This does not affect the commonmode noise cancellation properties of the circuit, but it should be noted that the frequency range for cancellation of noncommon mode noise sources (such as single beam drift) is always restricted to within the response time of the integrator. Reduction of the bandwidth of the Hobbs circuit has been reported previously [19] in measurements with pulsed quantum cascade lasers in order to enable the detector to respond to an average power rather than to individual pulses (bandwidths of 5 to $10 \mathrm{kHz}$ were used for a $15-\mathrm{kHz}$ pulse train, but still well above the sawtooth ramp frequency). Here however our motivation is different. By reducing the bandwidth below the sawtooth frequency, the AM absorption signal can now be demodulated from the linear output, which sits on a nominally $0 \mathrm{~V}$ background provided that the incident power levels are not changing too quickly relative to the slow response of the feedback. Since all common-mode background signals are nulled, the saturation limit of the lock-in amplifier gain is now determined by the depth of absorption and not the extent of the background signals as before.

Figure 1 shows the dual receiver Hobbs circuit from which the linear output is the input to the lock-in amplifier and the incident optical signals (intensity) on the signal and reference photo-diodes at the external modulation frequency $\omega$ are $\Delta I_{1}(v) e^{-\alpha C l} \cos (\omega t)$ and $\Delta I_{2}(v) \cos (\omega t)$ respectively. When the switch $\mathrm{S} 1$ is closed, the signal and reference signals combine at the summing junction before the trans-impedance amplifier stage to give the following signals at the output of the Lock-in amplifier referenced to $\omega$ :

$$
\begin{aligned}
& S_{\text {gas }}(v)=k\left[\Delta I_{1}(v) e^{-\alpha(v) C l}-r \Delta I_{2}(v)\right] \\
& S_{\text {nogas }}(v)=k\left[\Delta I_{1}(v)-r \Delta I_{2}(v)\right] \approx 0
\end{aligned}
$$

where $\mathrm{S}$ is the lock-in amplifier output signal from the linear output with and without gas, $\Delta I_{1}(v)$ and $\Delta I_{2}(v)$ are the optical intensity modulation (IM) amplitudes incident on the signal and reference photo-diodes respectively, $k$ is a simple scaling factor relating the optical IM signals to the output electronic signals from the lock-in amplifier and $r$ represents the photocurrent split ratio in the lower part of the autobalancing circuit controlled by the feedback loop to ensure that the no gas or baseline signal of equations (1) and (2) is nominally zero for the general case when $\Delta I_{1}(v) \neq \Delta I_{2}(v)$. 
The relative transmission can thus be calculated as:

$$
\frac{I_{\text {out }}}{I_{\text {in }}}=e^{-\alpha(v) C l}=1+\frac{S_{\text {gas }}(v)-S_{\text {nogas }}(v)}{k \Delta I_{1}(v)}
$$

where the normalisation factor, $k \Delta I_{1}(v)$, is easily obtained by opening the switch to record the output proportional to the signal photocurrent. Indeed, $k \Delta I_{1}(v)$ can be obtained, over the full frequency range of the scan, from the off-line data which can then be extrapolated to re-construct the $k \Delta I_{1}(v)$ over the frequency range of the absorption line.

Note that the "no gas signal" under ideal operation is nominally zero. However, in many practical arrangements there is a small off-set or background signal i.e. the no-gas signal is not zero and needs to be dealt with. In many applications it is not possible to take a no-gas measurement and fortunately it is not required. Similarly to the normalisation factor, the background signal can be obtained, over the full frequency range of the scan, from the off-line $S_{\text {gas }}$ data which can be extrapolated to re-construct the background signal over the frequency range of the absorption line.

Also note that, since equation (3) is independent of the power ratio $\Delta I_{1}(v) / \Delta I_{2}(v)$, it is possible for the beam power ratio to vary, as is common in a harsh environment, without affecting the normalised output line shape.

\section{EXPERIMENTAL SYSTEM}

A schematic of the experimental system used to evaluate the external modulation and electronic AM-nulling techniques is shown in Figure 2. The $10 \mathrm{~mW}$ OKI DFB laser (model: OL6109L-10B) is modulated with a $10 \mathrm{~Hz}$ sawtooth current ramp with an amplitude of $40 \mathrm{~mA}$. The operating wavelength of the laser diode was thermally tuned to coincide with the methane R-branch combination line at $1650.956 \mathrm{~nm}$ $\left(6057.10 \mathrm{~cm}^{-1}\right)$. The fibre-coupled laser diode output was split into four single mode fibre paths using a Sifam $1 \times 4$ splitter. One of these paths goes into a fibre ring resonator to facilitate wavelength referencing of the absorption signals. The operation of the fibre ring resonator is described in detail in [8].

The other two fibre outputs provide the two signals detected by the balanced photoreceiver. The first of these propagates through a Thorlabs FPC560 fibre polarisation control unit prior to a $2.5 \mathrm{~Gb} / \mathrm{s}$ SDL lithium niobate amplitude modulator (model: IOAP-MOD9082). The amplitude modulator uses a Mach-Zehnder interferometer to modulate the incoming signal using a sinusoidal voltage signal at a rate of $40 \mathrm{kHz}$. The modulated output signal is then collimated into a free space beam which interrogates a $5.5 \mathrm{~cm}$ fused silica gas cell, through which methane/nitrogen mixtures were flowed. The free space signal is detected by a Fermionics InGaAs photodiode with an active area diameter of $1.5 \mathrm{~mm}$. This diode was matched with a fibre-coupled InGaAs diode that detects the reference signal from the other output of the fibre splitter after passage through a variable optical attenuator to assist in attaining approximate signal balance. Table 1 give details of all the other components used in the circuit of Figure 1.

TABLE I

COMPONENTS USED IN THE CIRCUIT OF FIGURE 1

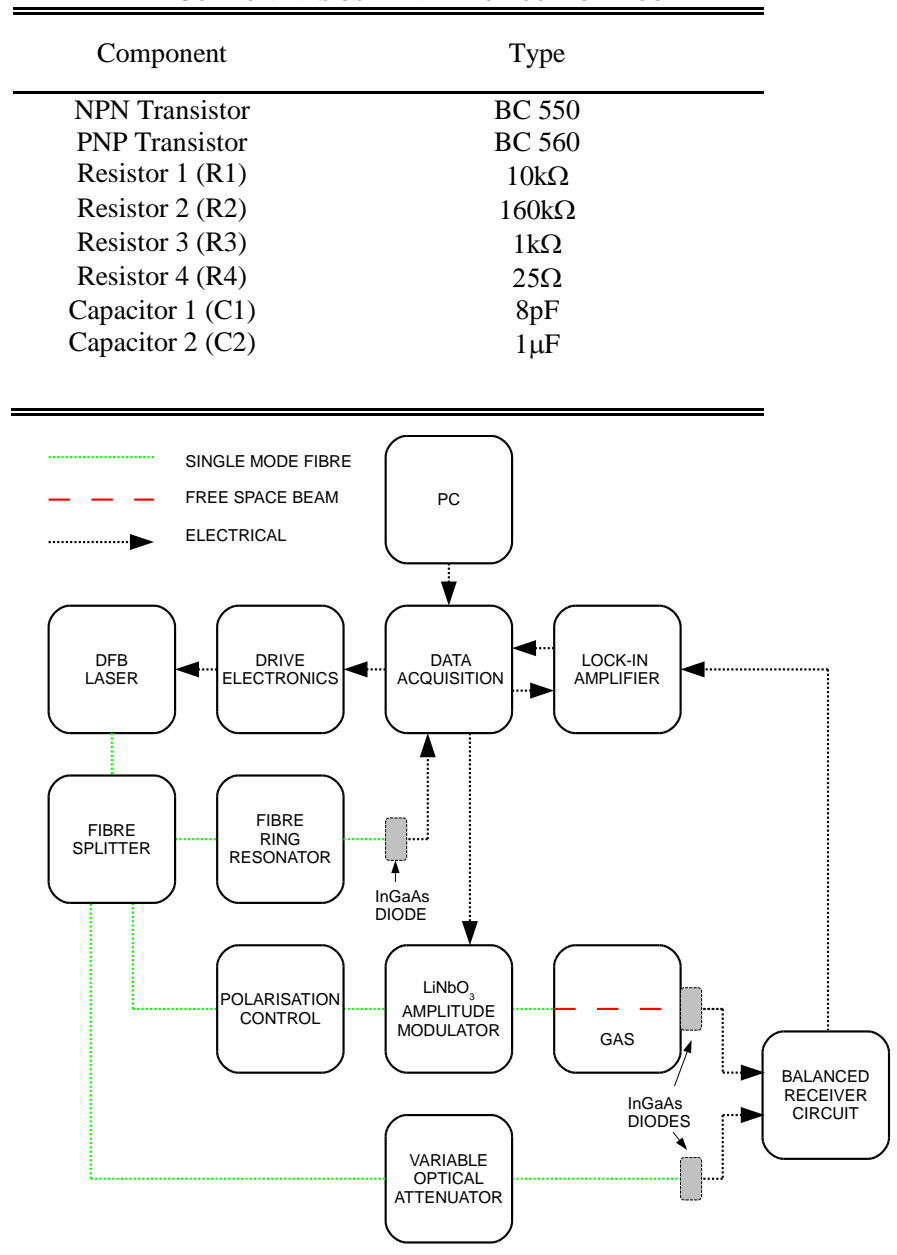

Fig. 2. Experimental system for line shape measurement with AM and background nulling

The response time of the integrator is determined by the values of $\mathrm{R} 2$ and $\mathrm{C} 2$ corresponding in this case to a $3 \mathrm{~dB}$ bandwidth of $0.1 \mathrm{~Hz}$. This means that essentially all noncommon mode noise sources above DC will appear unattenuated on the linear output. However, as usual with the BRD circuit, cancellation of common-mode noise (including the background AM signals arising from the intensity modulation) will occur over a very broad bandwidth, limited only by the transistor characteristics, i.e. gain-bandwidth, linearity, transistor matching, etc..

\section{EXPERIMENTAL RESULTS}

The effectiveness of the proposed technique was evaluated by flowing a mixture of $10 \%$ methane in nitrogen through the fused silica cell. The relatively strong absorbance allowed the line shapes from this technique to be compared to the equivalent direct detection line shapes with a high SNR. Figure 3 shows lock-in output signals at the fundamental frequency with the detection phase angle aligned with the modulation to maximise the recovered signal. The blue signal shows the normal transimpedance output (switch S1 in Figure 
1 open) whilst the red signal is the output with the switch, S1, closed allowing the background signals to be nulled. In this example the lock-in gain could be increased (sensitivity setting changed from $50 \mathrm{mV}$ to $20 \mathrm{mV}$ ) by switching on the nulling and the signals in the figure have been normalised by the sensitivity setting for comparison. The change in sensitivity setting is partly determined by the optical power and the relation between the amplified photoreceiver output and the lock-in overload limits for each discrete sensitivity setting. The RMS noise level was measured by taking a small off-line window and using MATLAB's 'std' (standard deviation) function which uses 1000 data points to make the calculation. For the signals shown in figure 3 and 4 the noise level in a non-absorbing section of the signal sweep is improved by a factor of $\sim 7$ (and also the SNR by $\sim 7$ since the absorption signals are of the same amplitude) by implementing the nulling. Later measurements of the noise levels observed in measurements of a $1000 \mathrm{ppm}$ methane in nitrogen mixture, show that when strong absorbance is not the limiting factor in the lock-in input overload level, the gain can be increased by a factor of 100 with a factor of $\sim 30$ reduction in the noise level (SNR increased by $\sim 30$ ) as a result of the background nulling with the modified Hobbs circuit.

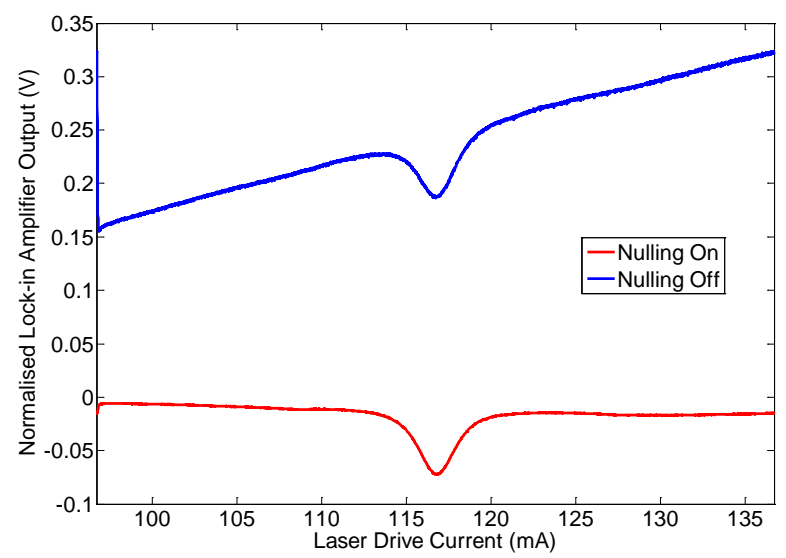

Fig. 3. Signals from the lock-in amplifier with and without backgroundnulling.

Figure 4 is a comparison between a direct detection signal, assumed to faithfully recover the true line shape, and the equivalent AM signals with and without background nulling. The unfiltered signals show clearly the reduction in noise levels for the nulled signals whilst the residuals do not show any clear structure that might indicate poor line shape recovery. Theoretical Voigt fits, based on the HITRAN08 database, were performed on these three signals to extract output readings for concentration and pressure. In this fitting procedure, the spectral parameters were calculated for the environmental conditions of temperature and pressure, and C and $\mathrm{P}$ were varied until a best fit was obtained using a least squares error methodology. The fit for the nulled external AM signal is shown in Figure 5, where the signal has been normalised using equation 3. The recovered concentration values for the normal AM and nulled AM absorption signals were within $0.3 \%$ of the direct detection output. The recovered values for pressure were within $1 \%$ of the direct output. These results clearly demonstrate that the modified version of the Hobbs circuit does not affect recovery of the absorption line shape.

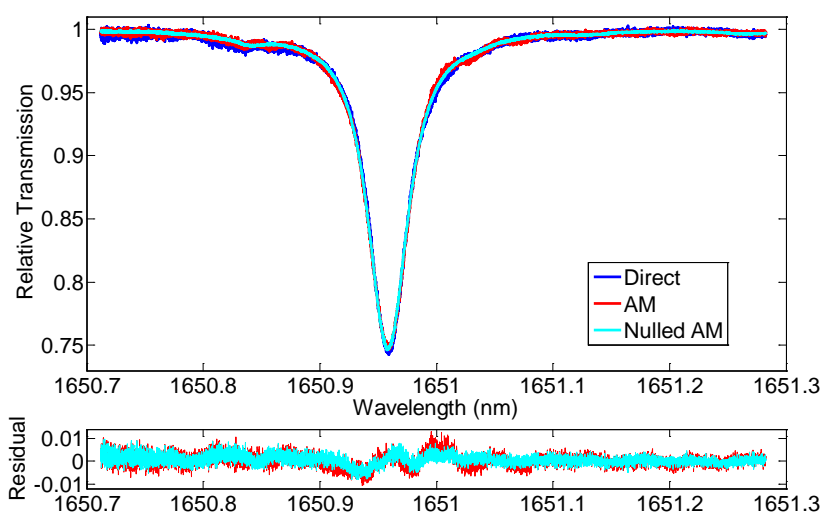

Fig. 4. Comparison of AM signals with that obtained from direct detection

A comparison of the achievable SNRs of various line shape recovery methods was also performed. A $1000 \mathrm{ppm}$ mixture of methane in nitrogen was used to provide the type of small signal that would benefit significantly from higher sensitivity recovery methods. Maintaining the same optical power levels and experimental states, measurements were taken using direct detection, external AM, background-nulled AM and WMS second harmonic signals by current modulation of the laser at $\mathrm{m}=2.2$. These methods are capable of directly recovering absorption line shapes with the exception of $2 \mathrm{f}$ WMS which was included to provide a comparison of the performance of these alternative techniques, in SNR terms, with a conventional wavelength modulation method.

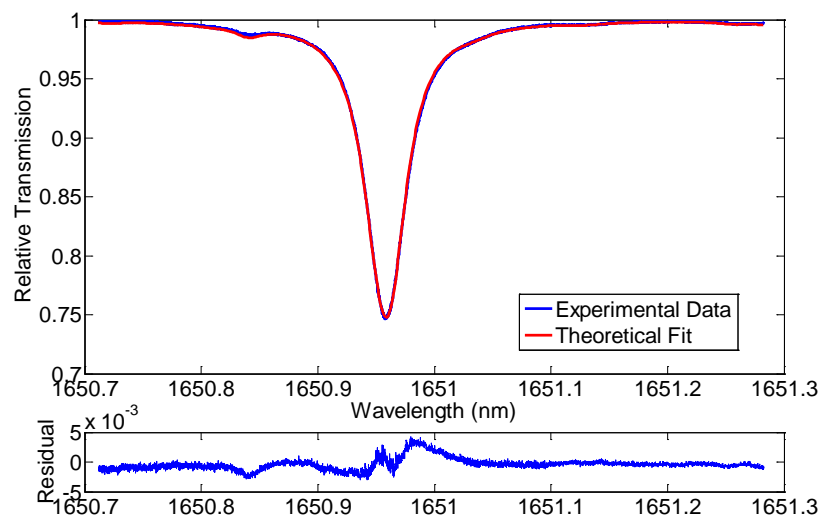

Fig. 5. Nulled AM signal compared with theoretical Voigt fit

Figure 6 compares all these signals for 1000 ppm methane. Clearly, the background nulled AM technique offers significant improvement in SNR terms over the non-nulled AM signals and indeed is comparable in SNR performance to the conventional $2 \mathrm{f}$ WMS approach. Measuring the RMS noise as previously from the standard deviation of the off-line data points, the average SNR for the direct, standard AM, nulled background AM and 2f WMS techniques respectively 
are 6.9, 6.8, 46.8 and 44 . The improvement in lock-in gain settings and SNR allowed by the nulled AM approach, depend on the optical signal power levels which ultimately determine the input voltage to the lock-in and its gain setting. Varying these parameters the optimum improvements we observed were a factor of 100 in terms of gain setting and a factor of 30 in SNR. In all cases the SNR of the background nulled AM signals were comparable to and in some cases greater than the 2f WMS signal.

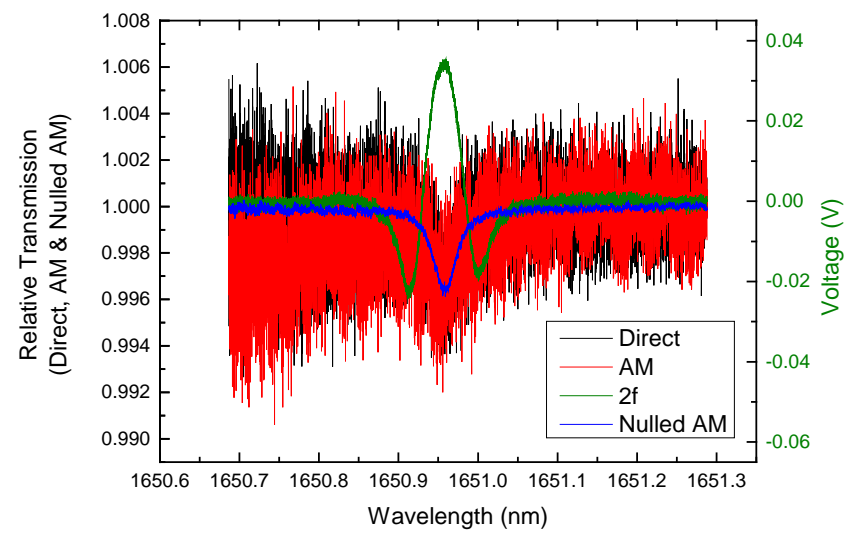

Fig. 6. Comparison of direct, external AM, nulled AM and second harmonic signals for 1000ppm methane.

\section{CONCLUSION}

We have demonstrated a method for electronic AM background nulling with external AM of the optical beam based on a simple modification to the Hobbs balanced photoreceiver circuit. The overall SNR is significantly improved over that obtained with direct detection or non-nulled AM techniques and is comparable to that of conventional second harmonic WMS. The important advantage of the technique over conventional $2 \mathrm{f}$ WMS is that normalisation and line shape recovery is straightforward and is independent of instrumentation scaling factors, or changes in laser characteristics and gas linewidth which affect the frequency modulation index applicable in WMS signal analysis.

\section{REFERENCES}

[1] B. Culshaw, G. Stewart, F. Dong, C. Tandy and D. Moodie, "Fibre Optic Techniques for Remote Spectroscopic Methane Detection - from Concept to System Realisation,” Sensors and Actuators B: Chemical, vol. 51, pp. 25-37, May 1998.

[2] G. Stewart, C. Tandy, D. Moodie, M. A. Morante and F. Dong, "Design of a Fibre Optic Multi-Point Sensor for Gas Detection," Sensors and Actuators B: Chemical, vol. 51, pp. 227-232, Aug. 1998.

[3] S. Schilt, L. Thevenaz and P. Robert "Wavelength modulation spectroscopy: Combined frequency and intensity laser modulation" Appl. Opt., vol. 42, no. 33, pp. 6728-6738, Nov. 2003.

[4] P. Kluczynski, A. M. Lindberg and O. Axner, "Characterization of Background Signals in Wavelength - Modulation Spectrometry in terms of a Fourier based Formalism,” Appl. Opt., vol. 40, no. 6, pp. 770-782, Feb. 2001.

[5] K. Duffin, A.J. McGettrick, W. Johnstone, G. Stewart and D.G. Moodie "Tuneable diode laser spectroscopy with wavelength modulation: a calibration-free approach to the recovery of absolute gas absorption lineshapes” J. Lightw. Technol., vol. 25, no. 10, pp. 3114-3125, Oct. 2007.

[6] A.J. McGettrick, K. Duffin, W. Johnstone, G. Stewart and D.G. Moodie "Tuneable diode laser spectroscopy with wavelength modulation: a phasor decomposition method for calibration free measurements of gas concentration and pressure" J. Lightw. Technol., vol. 26, no. 4, pp. 432440, Feb. 2008.

[7] W. Johnstone, A.J McGettrick, K. Duffin, A.Cheung and G. Stewart "Tuneable diode laser spectroscopy for industrial process applications: system characterisation in conventional and new approaches" IEEE Sensors Journal, vol. 8, no. 7, pp. 1079-1088, July 2008.

[8] A. J. McGettrick, W. Johnstone, R. Cunningham and J. Black, "Tunable diode laser spectroscopy: calibration free measurements of gas composition at elevated temperature and pressure", J. Lightw. Technol., vol. 27, no. 15, pp3150-3161, August 2009

[9] G. Stewart, W. Johnstone, J. R. P. Bain, K. Ruxton and K. Duffin "Recovery of Absolute Gas Absorption Line Shapes Using Tuneable Diode Laser Spectroscopy with Wavelength Modulation - Part 1: Theoretical Analysis” J. Lightw. Technol., vol. 29, no. 6, pp. 811-821, 2011

[10] J. R. P. Bain, W. Johnstone, K. Ruxton, G. Stewart, M. Lengden and K. Duffin "Recovery of Absolute Gas Absorption Line Shapes Using Tuneable Diode Laser Spectroscopy with Wavelength Modulation - Part 2: Experimental Investigation” J Lightw. Technol., vol. 29, no. 7, pp. 987-996, 2011

[11] A. L. Chakraborty, K Ruxton, W Johnstone, M Lengden and K Duffin "Elimination of residual amplitude modulation in tuneable diode laser wavelength modulation spectroscopy using an optical fiber delay line", Optics Express, vol. 17, no. 12, pp. 9602-9607, 2009.

[12] K. Sun, X Chao, R Sur, J Jeffries and R Hanson, "Wavelength modulation diode laser absorption spectroscopy for high-pressure gas sensing”, Appl. Phys. B, vol. 110, pp. 497-508, 2013.

[13] K. Sun, R. Sur, X. Chao, J. Jeffries, R. Hanson, R. Pummill, and K. Whitty, "TDL absorption sensors for gas temperature and concentrations in a high-pressure entrained-flow coal gasifier", Proceedings of the Combustion Institute, vol. 34, pp 3593-3601, 2013.

[14] C.S. Goldenstein, C. L. Strand, I. A. Schultz, K. Sun, J. B. Jeffries, and R. K. Hanson, "Fitting of calibration-free scanned-wavelengthmodulation spectroscopy spectra for determination of gas properties and absorption lineshapes” Appl Opt, vol. 53, no. 3, pp356-367, 2014.

[15] P. Wright, D. McCormick, K. Ozanyan, M. Johnson, J. Black, E. Fisher, A. Chighine, N. Polydorides, H. McCann, Y. Feng, K. Khan, P. Bastock, F. Jia, D. Hewak, J. Nilsson, M. Lengden, D. Wilson, I. Armstrong, T. Benoy and W. Johnstone, "Progress towards non-intrusive optical measurement of gas turbine exhaust species distributions", IEEE Aerospace 2015 Conference, pp. 1-14, Mar. 2015.

[16] P C Hobbs, "Ultra-sensitive laser measurements without tears", Appl. Opt., vol. 36, no. 4, pp. 903-920, Feb. 1997.

[17] X Zhu and D T Cassidy, "Electronic subtractor for trace gas detection with InGaAsP diode lasers”, Appl. Opt. vol. 34, no. 36, pp. 5546-5559, Dec. 1995.

[18] L Persson, F Andersson, M Andersson and S Svanberg, “Approach to optical interference fringes reduction in diode laser absorption spectroscopy”, Appl. Phys. B, vol. 87, pp. 523-530, 2007.

[19] D Sonnenfroh, W Rawlins, M Allen, C Gmachl, F Capasso, A Hutchinson, D Sivco, J Baillargeon, and A Cho, "Application of balanced detection to absorption measurements of trace gases with room-temperature, quasi-cw quantum-cascade lasers”, Appl. Opt. vol. 46, no. 6, pp. 812-820,2001.

James R.P. Bain received the B.Sc. (Hons.) degree in physics from the University of St. Andrews, St. Andrews, U.K., in 2006, the M.S.E.C.E. degree in electrical and computer engineering from the Georgia Institute of Technology, Atlanta, in 2007 and an Eng.D. degree in optics and photonics technologies at Rolls-Royce plc, London, U.K., and the Centre for Microsystems and Photonics, University of Strathclyde, Glasgow, U.K in 2012.

He was a Research Fellow within the Centre for Microsystems and Photonics until 2013. He is currently a Senior Project Manager at MSquared Lasers, Ltd., Glasgow. 
Michael Lengden received the M.Phys. degree in physics and the Ph.D. degree from the University of Manchester, Manchester, U.K., in 2001 and 2006, respectively, where he was engaged in stepwise excitation of atomic and molecular metastable states.

He was an Applications Engineer at the Laboratory Impex Systems, Ltd between 2006 and 2007 and a Research Fellow at the Centre for Microsystems and Photonics from 2007 to 2010. He is currently a Lecturer in the Electronics and Electrical Engineering Department, University of Strathclyde, Glasgow, U.K., where he is engaged in high-temperature and high-pressure gas composition measurements using tunable diode laser spectroscopy. His current research interests include new techniques in tunable diode laser spectroscopy for concentration and pressure measurements in harsh environments and miniaturized photoacoustic sensor development.

Dr. Lengden is a member of the Institute of Physics and the Optical Society of America.

George Stewart was awarded a BSc Degree with First Class Honours in 1974 and the PhD degree in 1979 for research on integrated optics, both from the University of Glasgow, Scotland, UK.

He continued research on integrated optics at the University of Glasgow until 1985 and then joined the University of Strathclyde, Glasgow, where his research has been in the areas of fibre optic components, evanescent wave devices, optical sensors, fibre lasers and DFB laser spectroscopy.

Professor Stewart is the author or co-author of 180 technical \& scientific papers in photonics and is a member of the Optical Society of America and the Institute of Physics.

Walter Johnstone received the B.Sc. (Hons.) degree in chemical and material sciences and the Ph.D. degree in laser physics from the University of Strathclyde, Glasgow, U.K., in 1977 and 1982, respectively.

From 1980 to 1986, he was at project engineering and senior technical management posts at Pilkington Optronics, Ltd. (now Thales) and Logitech, Ltd. He is currently the Vice Dean of Research at the University of Strathclyde, and a Professor of Photonic Systems in the Department of Electronic and Electrical Engineering. Since 1994, he has been a Director of OptoSci, Ltd., Glasgow, where he has been instrumental in bringing a number of photonics products to market. He has led to more than 170 technical publications and several patent applications. His current research interests include optical waveguide components, fiber lasers, distributed fiber sensing systems, and optical gas sensing. 